\title{
A Rapid and Sensitive UPLC-MS/MS Method for Determination of Remimazolam and Its Main Metabolite in Human Urine and Its Application in Clinical Urine Recovery Study
}

\author{
Ying Zhou ${ }^{1^{*}}$, Ming $\mathrm{Lu}^{2}$ and Ji Jiang ${ }^{2 *}$ \\ ${ }^{1}$ Department of Pharmacy, The First Affiliated Hospital of Nanchang University, Nanchang 330006, China \\ ${ }^{2}$ Clinical Pharmacology Research Center, Peking Union Medical College Hospital, \\ Peking Union Medical College and Chinese Academy of Medical Sciences, Beijing 100730, China
}

Received: 16 February 2017; accepted: 14 March 2017

\begin{abstract}
Remimazolam is a new chemical entity belonging to the benzodiazepine class of sedative drugs. A sensitive and rapid method based on ultra-performance liquid chromatography-tandem mass spectrometry (UPLC-MS/MS) has been developed and validated for the determination of remimazolam and its major carboxylic acid metabolite (M1) in human urine. Urine samples were prepared by dilution and analyzed using an isocratic chromatographic separation. Inter- and intra-batch results for remimazolam were within $10.7 \%$ for accuracy and $5.5 \%$ for precision, and for M1, within $5.8 \%$ for accuracy and $4.2 \%$ for precision, respectively. This study represents the first reported example for the quantification of remimazolam and its main metabolite in human urine. Furthermore, this method has been successfully applied for the urine recovery study of remimazolam in Chinese healthy subjects. Only about $0.01 \%$ of the administered remimazolam dose was eliminated in the urine over the $24 \mathrm{~h}$ period in the form of unchanged remimazolam, and more than $75.1 \%$ of the administered dose was eliminated in the form of M1. Remimazolam is excreted mainly in the form of M1 in urine after intravenous administration, and there is no excessive accumulation in vivo after administration of remimazolam.
\end{abstract}

Keywords: Remimazolam, metabolite, UPLC-MS/MS, urine recovery study, analytical method

\section{Introduction}

Remimazolam, which is designed to undergo rapid hydrolysis in the body by nonspecific tissue esterases to its pharmacologically inactive carboxylic acid metabolite (M1), is a new chemical entity belonging to the benzodiazepine class of sedative drugs [1]. Preclinical and clinical studies showed that remimazolam permitted a faster onset, a shorter duration of sedative action, and a more rapid recovery than currently available short-acting sedatives $[2,3]$. Therefore, because of its organ-independent metabolism and fast-acting onset and recovery, remimazolam appears to have potential advantages when used as an intravenous sedative agent for the induction and maintenance of anesthesia.

Remimazolam tosilate was approved by China Food and Drug Administration as an investigational new drug for the potential use in anesthesia in 2013 and is currently being evaluated in phase 3 trials. As we know, it is necessary to know about the route and extent of urinary excretion of a new drug, and the above information expresses the drug accumulation in vivo which is closely related to safety of the drug in human. Thus, it is necessary to develop a rapid and sensitive method to determine remimazolam and its main metabolite in human urine. To the best of our knowledge, there have been no other reports in the literature pertaining to the systematic determination of remimazolam and its main metabolite M1 in urine.

In this study, a sensitive and rapid method based on ultraperformance liquid chromatography-tandem mass spectrometry (UPLC-MS/MS) has been developed and validated for the determination of remimazolam and its major carboxylic acid metabolite (M1) in human urine [4]. The method was successfully applied for the pharmacokinetic studies of remimazolam to in-

* Author for correspondence: zyyiva@163.com, pk.frosh@gmail.com vestigate the route and extent of its urinary excretion in Chinese healthy subjects.

\section{Materials and methods}

2.1 Chemicals and reagents. Remimazolam tosilate and its internal standard $\left[{ }^{13} \mathrm{C}_{2}\right]$ remimazolam, carboxylic acid metabolite of remimazolam, and its internal standard $\left[{ }^{13} \mathrm{C}_{2}\right] \mathrm{M} 1$ (chemical structures are shown in Figure 1) were provided by Jiangsu Hengrui Medicine Co., Ltd. (Jiangsu, China). Methanol and acetonitrile (HPLC grade) was purchased from Burdick \& Jackson. Ammonium formate and formic acid (analytical grade) were supplied by Sigma-Aldrich, respectively. HPLC-grade water was obtained using a Milli-Q system (Millipore, Bedford, USA). Drug-free human urine was obtained from six different healthy subjects who were drug-free for at least 2 weeks.

2.2 Chromatographic and MS conditions. The chromatographic separation was carried out on an Acquity UPLC Core system (Waters Co., MA, USA). Isocratic elution was applied at a flow rate of $0.4 \mathrm{~mL} / \mathrm{min}$ using an Acquity UPLC CSH C $18(50 \mathrm{~mm} \times 2.1 \mathrm{~mm}, 1.7 \mu \mathrm{m})$ column at $25^{\circ} \mathrm{C}$. Mobile phase A was acetonitrile, and mobile phase B was water containing $10 \mathrm{mM}$ ammonium formate and $0.1 \%$ formic acid $(\mathrm{A}-\mathrm{B}=40: 60, \mathrm{v} / \mathrm{v})$. The run time was $1.5 \mathrm{~min}$, and the injection volume was $7.5 \mu \mathrm{L}$.

Mass spectrometric analysis was performed using Xevo-TQS triple quadrupole mass spectrometer equipped with an electrospray ionization (ESI) source in the positive mode (Waters Co., MA, USA). The detection was operated in the multiple reaction monitoring (MRM) mode, and the MRM transitions of remimazolam and its IS were $m / z 439 \rightarrow 362$ and $m / z 444 \rightarrow 412$, and those of M1 and its IS were $m / z 425 \rightarrow 407$ and $m / z 430 \rightarrow 412$, respectively. The ionization source conditions were cone voltage,

This is an open-access article distributed under the terms of the Creative Commons Attribution-NonCommercial 4.0 International License (https://creativecommons.org/licenses/by-nc/4.0/), which permits unrestricted use, distribution, and reproduction in any medium for non-commercial purposes, provided the original author and source are credited, a link to the CC License is provided, and changes - if any - are indicated. 


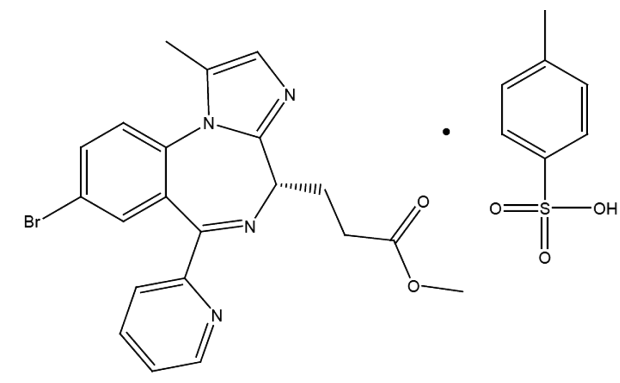

a

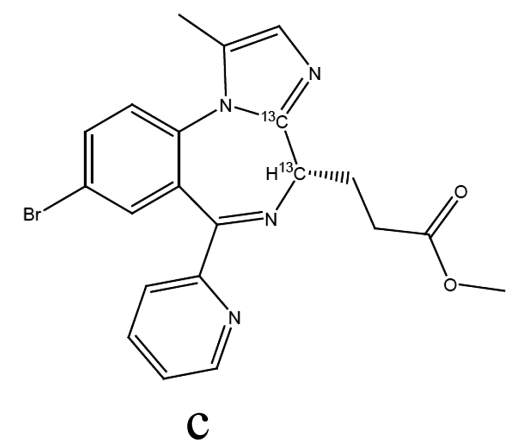

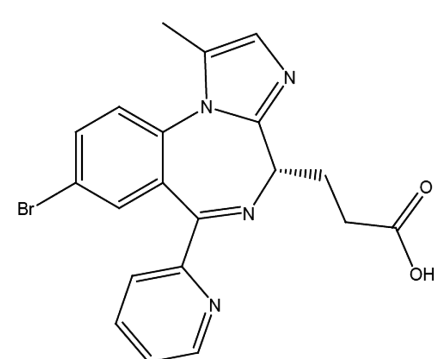

b

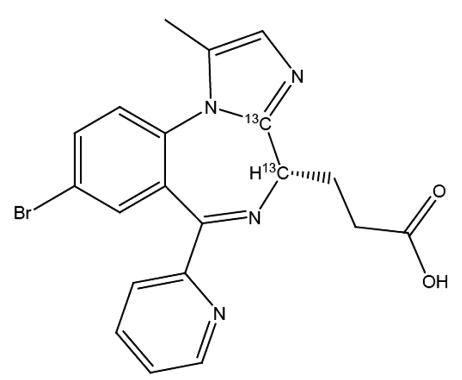

d

Figure 1. Chemical structures of remimazolam tosilate (a), M1 (b), $\left[{ }^{13} \mathrm{C}_{2}\right]$ remimazolam (c), and $\left[{ }^{13} \mathrm{C}_{2}\right] \mathrm{M} 1$ (d)

$45 \mathrm{~V}$; capillary voltage, $3 \mathrm{kV}$; source temperature, $150{ }^{\circ} \mathrm{C}$; and desolvation temperature, $600{ }^{\circ} \mathrm{C}$. The optimized collision energy of remimazolam and its IS, and M1 and its IS were $30 \mathrm{~V}$ and $30 \mathrm{~V}$, and $20 \mathrm{~V}$ and $18 \mathrm{~V}$, respectively. The cone and desolvation gas flow rates were 150 and $1000 \mathrm{~L} / \mathrm{h}$, respectively. Data acquisition and processing were performed using MassLynx software (version 4.1).

2.3 Preparation of calibration standard (CS) and quality control (QC) samples. Stock solutions of remimazolam and M1 for CS and QC were prepared separately in methanol to a final concentration of $1 \mathrm{mg} / \mathrm{mL}$ for each analyte, and stock solutions of their IS $(1 \mathrm{mg} / \mathrm{mL})$ were also prepared separately in methanol. These stock solutions were further diluted to yield working solutions at several concentration levels.

Calibration standards and QC samples in urine were prepared by diluting corresponding working solutions with drugfree human urine, respectively. The final concentrations of calibration standards of remimazolam were from 0.5 to $1000 \mathrm{ng} /$ $\mathrm{mL}$ and those of M1 were from 20 to $40,000 \mathrm{ng} / \mathrm{mL}$. The final concentrations of QC samples for the evaluation of intra- and inter-batch precision and accuracy, matrix effect, and stability were $1.5,30$, and $750 \mathrm{ng} / \mathrm{mL}$ for remimazolam and 60,1200 , and $30,000 \mathrm{ng} / \mathrm{mL}$ for $\mathrm{M} 1$, respectively. IS working solutions (100 ng/mL for remimazolam and $80 \mathrm{ng} / \mathrm{mL}$ for M1) were prepared with mobile phase $(\mathrm{A}-\mathrm{B}=40: 60, \mathrm{v} / \mathrm{v})$. All the solutions were stored at $-30{ }^{\circ} \mathrm{C}$ and brought to room temperature $\left(25^{\circ} \mathrm{C}\right)$ before use. Urine samples were stored at $-70{ }^{\circ} \mathrm{C}$.

2.4 Sample preparation. Urine samples were pretreated by dilution procedure with different dilution factors. Urine samples for the quantitative determination of remimazolam were prepared by diluting $50 \mu \mathrm{L}$ of urine sample with $250 \mu \mathrm{L}$ of IS working solution $\left(100 \mathrm{ng} / \mathrm{mL}\left[{ }^{13} \mathrm{C}_{2}\right]\right.$ remimazolam in mobile phase). Urine samples for the quantitative determination of M1 were prepared by diluting $50 \mu \mathrm{L}$ of urine sample with $950 \mu \mathrm{L}$ of mobile phase. Then, $30 \mu \mathrm{L}$ of the diluted urine sample was combined with $270 \mu \mathrm{L}$ of IS working solution (80 ng/mL $\left[{ }^{13} \mathrm{C}_{2}\right] \mathrm{M} 1$ in mobile phase). Following vortex mixing (for $1 \mathrm{~min}$ ) and centrifugation (for 5 min at $16,242 \times g$ ) to settle any particulate matter, $7.5 \mu \mathrm{L}$ of the supernatant was injected to the UPLC-MS/MS system.
2.5 Method validation. The method was validated for selectivity, sensitivity, linearity, precision and accuracy, matrix effects, and stability according to the US Food and Drug Administration (FDA) (2013) [5] and China Food and Drug Administration (CFDA) guidelines (2005) [6] for the validation of bioanalytical methods.

The selectivity of this method was evaluated by comparison of the chromatograms of remimazolam and M1 at the LLOQ to those of six individual human blank urine samples.

Calibration standards in human urine were prepared and analyzed in three independent runs. The relative standard deviation (RSD) was calculated for all slopes of calibration curves. The LLOQ is defined as the lowest concentration on the calibration curve. Intra- and inter-batch precision and accuracy were determined by measuring the concentrations of analyte in urine in five replicates of QC samples at low, medium, and high concentrations for three separate batches.

Matrix effects were evaluated to identify any suppression or enhancement of signal from an interfering substance around the retention time of remimazolam and M1. The matrix effects were investigated at three levels $(1.5,30$, and $750 \mathrm{ng} / \mathrm{mL}$ for remimazolam; 60,1200 , and $30,000 \mathrm{ng} / \mathrm{mL}$ for M1) by comparing the peak areas of post-extraction blank urine samples from six different subjects spiked with analyte with that of the standards in mobile phase at the same concentration level. The inter-subject variability of matrix effects at each concentration level should be less than $15 \%$ [7-9].

The stabilities of remimazolam and M1 in biological matrices and in working solution at different storage conditions were evaluated as follows: The short-term stability of analytes in human urine was assessed after $24 \mathrm{~h}$ of storage at room temperature. The long-term stabilities of analytes in human urine and in working solution were assessed after 291 days of storage in a freezer at $-70{ }^{\circ} \mathrm{C}$ and 44 days of storage in a freezer at $-30{ }^{\circ} \mathrm{C}$, respectively. The stability of analytes was assessed after three freeze-thaw cycles $\left(-70^{\circ} \mathrm{C}\right.$ to room temperature). The stability of analytes in extracts was also tested after $24 \mathrm{~h}$ at $10{ }^{\circ} \mathrm{C}$.

2.6 Application of the method to urine recovery study. The validated method was applied to quantify urine 
concentrations of remimazolam and M1 in a urine recovery study. Thirty-six healthy subjects were enrolled in this single ascending-dose study (doses ranging from $0.10 \mathrm{mg} / \mathrm{kg}$ to $0.35 \mathrm{mg} / \mathrm{kg}$ ) of remimazolam administered as a 1 minute intravenous (IV) injection. Urine samples were continuously collected up to $24 \mathrm{~h}$ prior to and in the following intervals: 0 to 4,4 to 8,8 to 12 , and 12 to $24 \mathrm{~h}$. Aliquots of urine samples were stored at $-70{ }^{\circ} \mathrm{C}$ until analysis. The study was conducted in accordance with International Conference on Harmonization Good Clinical Practice guidelines, all applicable subject privacy requirements, and the ethical principles outlined in the Declaration of Helsinki. The study was approved by the Ethics Committee of Peking Union Medical College Hospital, and all subjects signed the informed consent form before the study.

\section{Results}

\subsection{Validation procedure}

3.1.1 Selectivity and sensitivity. No peaks interfering were observed at the retention times of remimazolam, M1, and the internal standards in the blank urine samples. Typical MRM chromatograms obtained from blank urine, blank urine spiked with remimazolam or M1 (LLOQ) and their relative internal standards, and a subject's urine sample obtained at interval of 0-4 h after 1 minute IV administration of $0.35 \mathrm{mg} / \mathrm{kg}$ remimazolam tosilate injection are shown in Figure 2.

3.1.2 Linearity, precision, and accuracy. Calibration curves ranging from 0.5 to $1000 \mathrm{ng} / \mathrm{mL}$ for remimazolam and from 20 to $40,000 \mathrm{ng} / \mathrm{mL}$ for M1, respectively, were established. Calibration curves were regressed using linear equation with a weighting factor of $1 / x^{2}$. Correlation coefficients of all calibration curves were more than 0.99 . The deviations of back-calculated concentrations of calibration standards from their nominal values were between $-0.5 \%$ and $1.6 \%$ for LLOQ, and between $-6.5 \%$ and $4.4 \%$ for all other calibration levels.

Precision and accuracy values were determined on three different batches by measuring five replicates of QC samples at three concentration levels. The results are listed in Table 1. It indicated that the method provided adequate precision and accuracy for the determination of two analytes since RSD\% was $<15 \%$ for all the investigated concentrations and $\mathrm{RE} \%$ was within $\pm 15 \%$.

3.1.3 Matrix effects. Matrix effects and inter-subject variability data from three concentrations of QC urine samples are summarized in Table 2. The inter-subject variabilities were no more than $10.2 \%$ for both analytes. This indicated little or no difference in ionization efficiency of remimazolam and M1 from different urine lots. The urine assay was not tested for recovery as the sample preparation involved a simple dilution step only.

3.1.4 Stability. The stability tests of the analytes were designed to cover expected conditions of handling of clinical samples. The results demonstrated that remimazolam and M1 were stable in

Table 2. Results of matrix effects of remimazolam and M1 in human urine $(n=6)$

\begin{tabular}{lcc}
\hline Compound & $\begin{array}{c}\text { Nominal Concentration } \\
(\mathrm{ng} / \mathrm{mL})\end{array}$ & $\begin{array}{c}\text { Matrix effects\% } \\
\left.\text { (inter-subject variability\% } \%{ }^{a}\right)\end{array}$ \\
\hline Remimazolam & 1.50 & $84.8(3.6)$ \\
& 30.0 & $89.7(2.9)$ \\
M1 & 750 & $88.5(1.7)$ \\
& 60.0 & $110.7(4.7)$ \\
& 1200 & $108.3(3.0)$ \\
${ }^{a}$ Expressed as RSD \%. & & \\
\hline
\end{tabular}

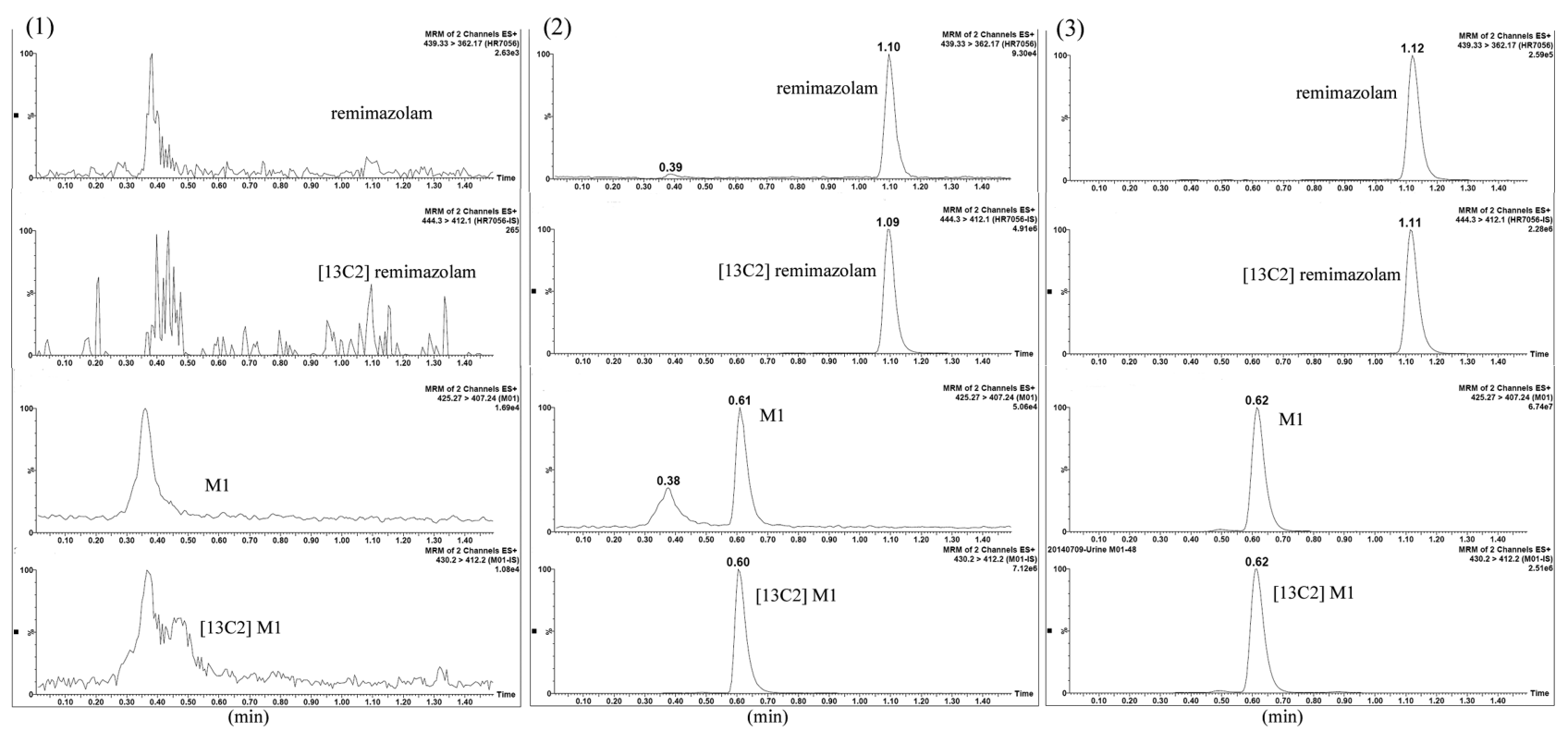

Figure 2. Representative MRM chromatograms of remimazolam, M1, $\left[{ }^{13} \mathrm{C}_{2}\right]$ remimazolam, and $\left[{ }^{13} \mathrm{C}_{2}\right] \mathrm{M} 1$ : (1) blank urine sample, (2) blank urine sample spiked with two analytes at LLOQ and two internal standards (100 ng/mL for remimazolam and $80 \mathrm{ng} / \mathrm{mL}$ for M1), and (3) urine sample collected from a healthy subject at interval of $0-4 \mathrm{~h}$ after 1 minute IV administration of $0.35 \mathrm{mg} / \mathrm{kg}$ remimazolam tosilate injection

Table 1. Accuracy and precision of intra- and inter-batch for the detection of remimazolam and M1 in human urine $(n=5)$

\begin{tabular}{lccccc}
\hline Compound & $\begin{array}{c}\text { Nominal concentration } \\
(\mathrm{ng} / \mathrm{mL})\end{array}$ & $\begin{array}{c}\text { Intra-batch accuracy } \\
(\%)\end{array}$ & $\begin{array}{c}\text { Inter-batch accuracy } \\
(\%)\end{array}$ & $\begin{array}{c}\text { Intra-batch precision } \\
(\text { RSD } \%)\end{array}$ & $\begin{array}{c}\text { Inter-batch precision } \\
(\text { RSD } \%)\end{array}$ \\
\hline Remimazolam & 1.50 & 94.0 & 89.3 & 3.6 & 3.3 \\
& 30.0 & 103.3 & 97.3 & 3.3 & 5.5 \\
M1 & 750 & 97.9 & 95.9 & 3.6 & 3.0 \\
& 60.0 & 97.3 & 96.3 & 3.8 & 4.2 \\
& 1200 & 95.0 & 98.2 & 2.8 & 3.1 \\
\hline
\end{tabular}


Table 3. Results of stability of remimazolam and M1 under different storage conditions $(n=5)$

\begin{tabular}{|c|c|c|c|c|}
\hline \multirow[t]{2}{*}{ Storage condition } & \multicolumn{2}{|c|}{ Nominal concentration $(\mathrm{ng} / \mathrm{mL})$} & \multicolumn{2}{|c|}{ Measured mean concentration $(\mathrm{RE} \%)(\mathrm{ng} / \mathrm{mL})$} \\
\hline & Remimazolam & M1 & Remimazolam & M1 \\
\hline \multirow[t]{3}{*}{ Short-term stability $^{a}$} & 1.50 & 60.0 & $1.52(1.7)$ & $64.7(7.8)$ \\
\hline & 30.0 & 1200 & $28.8(-4.2)$ & $1259(4.9)$ \\
\hline & 750 & 30,000 & $736(-1.8)$ & $30,757(2.5)$ \\
\hline \multirow[t]{3}{*}{ Long-term stability $^{b}$} & 1.50 & 60.0 & $1.65(10.1)$ & $55.1(-8.1)$ \\
\hline & 30.0 & 1200 & $30.7(2.5)$ & $1140(-5.0)$ \\
\hline & 750 & 30,000 & $763(1.7)$ & $27,937(-6.9)$ \\
\hline \multirow[t]{3}{*}{ Freeze-thaw stability ${ }^{c}$} & 1.50 & 60.0 & $1.45(-3.4)$ & $65.1(8.5)$ \\
\hline & 30.0 & 1200 & $32.7(9.0)$ & $1275(6.3)$ \\
\hline & 750 & 30,000 & $823(9.8)$ & $30,795(2.7)$ \\
\hline \multirow[t]{3}{*}{ Auto-sampler stability $^{d}$} & 1.50 & 60.0 & $1.68(12.1)$ & $65.0(8.4)$ \\
\hline & 30.0 & 1200 & $29.6(-1.5)$ & $1312(9.3)$ \\
\hline & 750 & 30,000 & $738(-1.5)$ & $32,211(7.4)$ \\
\hline $\begin{array}{l}{ }^{a} \text { Stored at room temp } \\
{ }^{b} \text { Stored at }-70{ }^{\circ} \mathrm{C} \text { for } \\
{ }^{c} \text { After three freeze-th } \\
{ }^{d} \text { Kept at } 10^{\circ} \mathrm{C} \text { for } 24\end{array}$ & for $24 \mathrm{~h}$. & & & \\
\hline
\end{tabular}

Table 4. Urinary excretions of remimazolam and M1 in Chinese healthy subjects after 1 minute IV administration of remimazolam tosilate injection of different doses $(n=6)$

\begin{tabular}{|c|c|c|c|c|c|c|}
\hline Collection interval (h) & $0.10 \mathrm{mg} / \mathrm{kg}$ & $0.15 \mathrm{mg} / \mathrm{kg}$ & $0.20 \mathrm{mg} / \mathrm{kg}$ & $0.25 \mathrm{mg} / \mathrm{kg}$ & $0.30 \mathrm{mg} / \mathrm{kg}$ & $0.35 \mathrm{mg} / \mathrm{kg}$ \\
\hline \multicolumn{7}{|l|}{ Remimazolam } \\
\hline $0-4$ & $0.01 \pm 0.01$ & $0.01 \pm 0.01$ & $0.01 \pm 0.01$ & $0.01 \pm 0.01$ & $0.01 \pm 0.01$ & $0.01 \pm 0.01$ \\
\hline $4-8$ & - & - & - & - & - & - \\
\hline $12-24$ & - & - & - & - & - & - \\
\hline Total & $0.01 \pm 0.01$ & $0.01 \pm 0.01$ & $0.01 \pm 0.01$ & $0.01 \pm 0.01$ & $0.01 \pm 0.01$ & $0.01 \pm 0.01$ \\
\hline \multicolumn{7}{|l|}{ M1 } \\
\hline $0-4$ & $54.7 \pm 8.91$ & $55.0 \pm 5.58$ & $58.5 \pm 8.90$ & $58.3 \pm 7.05$ & $63.4 \pm 3.17$ & $50.6 \pm 10.5$ \\
\hline $4-8$ & $16.4 \pm 4.23$ & $17.1 \pm 5.86$ & $19.6 \pm 4.61$ & $19.2 \pm 5.21$ & $19.4 \pm 3.05$ & $17.0 \pm 2.75$ \\
\hline $8-12$ & $5.70 \pm 2.10$ & $5.74 \pm 3.38$ & $6.47 \pm 1.76$ & $5.07 \pm 2.45$ & $4.79 \pm 2.34$ & $5.66 \pm 2.41$ \\
\hline $12-24$ & $2.17 \pm 1.19$ & $1.97 \pm 0.83$ & $1.98 \pm 0.49$ & $1.95 \pm 1.12$ & $1.87 \pm 0.30$ & $1.83 \pm 0.98$ \\
\hline Total & $79.0 \pm 7.98$ & $79.8 \pm 13.1$ & $86.5 \pm 6.04$ & $84.5 \pm 7.05$ & $89.5 \pm 4.07$ & $75.1 \pm 9.16$ \\
\hline
\end{tabular}

human urine after three freeze-thaw cycles, at room temperature and $10{ }^{\circ} \mathrm{C}$ in autosampler post-extraction for $24 \mathrm{~h}$ and in a freezer set to $-70{ }^{\circ} \mathrm{C}$ for 291 days. Working solutions of remimazolam and $\mathrm{M} 1$ stored at $-30^{\circ} \mathrm{C}$ for 44 days also showed good stability. The stability results are summarized in Table 3 .

3.2 Application of the method to urine recovery study. The present UPLC-MS/MS method was successfully applied to investigate the urine pharmacokinetic profile of remimazolam after 1 minute IV administration of remimazolam tosilate injection to Chinese healthy subjects. Table 4 shows the urinary excretion of remimazolam and M1 in subjects treated with different doses of remimazolam tosilate injection. Only about $0.01 \%$ of the administered remimazolam dose was eliminated in the urine over the $24 \mathrm{~h}$ period in the form of unchanged remimazolam, and more than $75.1 \%$ of the administered dose was eliminated in the form of M1. In the meanwhile, the majority of remimazolam and M1 were excreted during the 0 to $4 \mathrm{~h}$ collection interval (about $67.4 \%$ of total amount of remimazolam and M1 excreted in the urine). The maximum rate of remimazolam excretion occurred during the 0 to $4 \mathrm{~h}$ collection interval.

\section{Discussion}

4.1 Sample preparation. Direct dilution was used to prepare urine samples because urine samples are cleaner compared with plasma samples and no interference was found in the blank urine sample. Urine samples were diluted with different dilution factors to bring concentrations to a linear range.

4.2 UPLC-MS/MS optimization. An UPLC-MS/MS method for the detection of remimazolam and M1 in human urine was developed based on the previous study. The $[\mathrm{M}+\mathrm{H}]^{+}$ were chosen as the precursor ions for all analytes, and the fragment ions having only about $20 \%$ of the abundance of that of the molecular ions were chosen as the precursor ions for $\left[{ }^{13} \mathrm{C}_{2}\right]$ remimazolam and $\left[{ }^{13} \mathrm{C}_{2}\right] \mathrm{M} 1$ in order to avoid the "crosstalk" effect caused by isotopic peak of bromine. Also, we chose the product ions based on the most abundant peak, cleaner chromatogram, and higher $\mathrm{S} / \mathrm{N}$ ratio.

During the development of chromatographic conditions, mobile phase systems with different $\mathrm{pH}$ values and different organic solvents, such as methanol and acetonitrile, were investigated. When acetonitrile-water containing $10 \mathrm{mM}$ ammonium formate and $0.1 \%$ formic acid (40:60, v/v) pumped at a flow rate of $0.4 \mathrm{~mL} / \mathrm{min}$ at $25^{\circ} \mathrm{C}$ was used as eluent, a good chromatographic profile and sensitivity were achieved. After careful comparison of several columns, an Acquity UPLC CSH $\mathrm{C}_{18}(50 \mathrm{~mm} \times 2.1 \mathrm{~mm}, 1.7 \mu \mathrm{m})$ column was proved to be more suitable as it can provide sufficient retention and better peak shape for analytes.

4.3 Urine recovery study. Urine recovery study showed that only about $0.01 \%$ of the administered remimazolam dose was eliminated in the urine over the $24 \mathrm{~h}$ period in the form of unchanged remimazolam, and more than $75.1 \%$ of the administered dose was eliminated in the form of M1. The rest $25 \%$ of the initial dose were eliminated in the forms of oxidated and glucuronide metabolites [10]. The maximum rate of remimazolam excretion occurred during the 0 to $4 \mathrm{~h}$ collection interval. Based on these data, it can be concluded that remimazolam is excreted mainly in the form of M1 in urine after intravenous administration and there is no excessive accumulation in vivo after administration of remimazolam.

\section{Conclusion}

A simple, sensitive, and specific UPLC-MS/MS method has been developed and validated for the determination of 
remimazolam and its main metabolite M1 in human urine, which requires a very low sample volume and takes a very short time. Based on the results of the validation of the current study, it is envisaged that this simple, highly sensitive, and specific method for determination of remimazolam and M1 in human urine could apply a better understanding of the urinary excretion of remimazolam, which gives important information about accumulation and safety in Chinese healthy subjects after intravenous administration of remimazolam. This study represents the first reported example for the quantification of remimazolam and its main metabolite in human urine and has been successfully applied for the PK study of remimazolam to investigate the route and extent of its urinary excretion in Chinese healthy subjects.

\section{Ethical approval}

The study was conducted in accordance with International Conference on Harmonization Good Clinical Practice guidelines, all applicable subject privacy requirements, and the ethical principles outlined in the Declaration of Helsinki.

\section{Informed consent}

Informed consent was obtained from all individual participants included in the study.
Acknowledgments. The author thanks all of the subjects who participated in the clinical study.

This study was financially supported by Jiangsu Hengrui Medicine Co., Ltd. This work was also supported by a grant from the National Program on Key Research Project of New Drug Innovation (No. 2012ZX09303006-002).

\section{References}

1. Kilpatrick, G. J.; Mclntyre, M. S.; Cox, R. F.; Stafford, J. A.; Pacofsky, G. J.; Lovell, G. G.; et al. Anesthesiology 2007, 1, 60 .

2. Rogers, W. K.; McDowell, T. S. IDrugs 2010, 12, 929.

3. Upton, R. N.; Somogyi, A. A.; Martinez, A. M.; Colvill, J.; Grant, C. Br J Anaesth 2010, 6, 798.

4. Zhou, Y.; Wang, H.; Jiang, J.; Hu, P. J Chromatogr B Analyt Technol Biomed Life Sci 2015, 976-977, 78.

5. US FDA, Guidance for Industry Bioanalytical Method Validation, 2013 , www.fda.gov/downloads/drugs/guidancecomplianceregulatoryinformation/guidances/ ucm368107.pdf, Accessed 22 Aug 2016.

6. China Food and Drug Administration, Guideline on clinical pharmacokinetic study of drugs, 2005, www.sfda.gov.cn/directory/web/WS01/ images/u6Rp9KpzuB2bSy0qm0+ravwabRp9HQvr+8vMr11ri1vNSt1PIucGRm.pdf, Accessed 22 Aug 2016.

7. Taylor, P. J. Clin Biochem 2005, 38, 328

8. Matuszewski, B. K.; Constanzer, M. L.; Chavez-Eng, C. M. Anal Chem 2003, 75, 3019 .

9. Matuszewski, B. K. J Chromatogr B Analyt Technol Biomed Life Sci 2006, 830, 293.

10. Zhou, Y., Hu, P., Jiang, J. J Pharm Biomed Anal 2017, 137, 78. 